\title{
COMPLETIONS OF UNIFORM CONVERGENCE SPACES ${ }^{1}$
}

\author{
G. D. RICHARDSON
}

Abstract. H. J. Biesterfeldt has shown that a uniform convergence space which satisfies the completion axiom has a completion. In the present paper, we show that every uniform convergence space has a completion. Furthermore, if the uniform convergence space is Hausdorff and satisfies the completion axiom, then it has a Hausdorff completion, which reduces to the Bourbaki completion for uniform spaces. Finally, a uniqueness theorem is obtained.

1. Introduction. Cook and Fischer introduced the notion of a uniform convergence space, which is a generalization of a uniform space. Each uniform convergence space induces a "limesräume" [4], which is a generalization of a topological space.

Biesterfeldt [1] has shown that a uniform convergence space which satisfies the completion axiom has a completion. By slightly modifying Biesterfeldt's construction, we show that every uniform convergence space has a completion. Furthermore, by introducing an equivalence relation, we show that each Hausdorff uniform convergence space which satisfies the completion axiom has a Hausdorff completion. Finally, a uniqueness theorem among Hausdorff completions is obtained.

2. Completions. The reader is asked to refer to [3] and [4] for basic definitions and notation not given here. We will abbreviate the terms "uniform convergence space (structure)" and "completion axiom" by "u.c.s." and "c.a.", respectively.

Definition 2.1. $B$ is called a base for a u.c.s. $I$ for $X$ if (1) $B C I$ and (2) $\Phi \in I$ implies there exists a $\psi \in B$ such that $\Phi$ is finer than $\psi$, i.e. $\Phi \geqq \psi$.

Hence we have the following:

Proposition 2.2. A collection $B$ of filters on $X \times X$ is a base for some u.c.s. iff the following hold:

Received by the editors August 20, 1970.

AMS 1969 subject classifications. Primary 5422, 5410; Secondary 5420.

Key words and phrases. Uniform convergence space, completion, Cauchy filter, uniform isomorphism.

1 This is a portion of the author's thesis at North Carolina State University. The author sincerely thanks Professors P. A. Nickel and R. E. Chandler for helpful suggestions; also the Ford Foundation for partial support.

Copyright (c) 1971, American Mathematical Society 
(1) $[\Delta]$ is finer than some $\Phi$ in $B$.

(2) $\Phi, \psi$ in $B$ implies $\Phi \wedge \psi \geqq \Gamma$ for some $\Gamma$ in $B$.

(3) $\Phi$ in $B$ implies $\Phi^{-1} \geqq \psi$ for some $\psi$ in $B$.

(4) $\Phi, \psi$ in $B$ such that $\Phi \circ \psi$ exists, then $\Phi \circ \psi \geqq \Gamma$ for some $\Gamma$ in $B$.

Let $(X, I)$ be a u.c.s. We denote by $X^{\prime}$ the collection of all Cauchy filters on $X, A^{\prime}=\left\{(F, G): \mathcal{F}, g \in X^{\prime}\right.$ and $F \times G \subset A$ for some $F \in \mathcal{F}$, $G \in \mathcal{G}\}$, and $\Phi^{\prime}$ denotes the filter on $X^{\prime} \times X^{\prime}$ generated by $\left\{A^{\prime}: A \in \Phi\right\}$. We call $\mathcal{F}$ and $\mathcal{G} A$-near if $(\mathcal{F}, \mathcal{G})$ belongs to $A^{\prime}$.

Biesterfeldt could have avoided using the c.a. where he did, but his construction would still have fallen short since the diagonal filter would not have been in his completion without using the c.a. However, since the proofs here vary only slightly from those of [1], we will only outline the steps for a completion of $(X, I)$.

Using Proposition 2.2, we can show that $B=\left\{\Phi^{\prime} \wedge\left[\Delta_{1}\right]: \Phi \in I\right\}$ is a base for a u.c.s. $I^{\prime}$ for $X^{\prime}$, where $\Delta_{1}$ is the diagonal of $X^{\prime} \times X^{\prime}$. The u.c.s. $\left(X^{\prime}, I^{\prime}\right)$ is our candidate for a "completion" of $(X, I)$. Define $i: X \rightarrow X^{\prime}$ by $i(x)=\phi(x)$, where $\phi(x)$ denotes the filter on $X$ generated by the set $\{x\}$. It can be shown that $i$ is a uniform isomorphism from $(X, I)$ onto a dense subspace of $\left(X^{\prime}, I^{\prime}\right)$.

TheOREM 2.3. The u.c.s. $\left(X^{\prime}, I^{\prime}\right)$ is a completion of $(X, I)$.

We need only to show that $\left(X^{\prime}, I^{\prime}\right)$ is complete. This will follow from our succeeding lemmas. Note, we do not need the c.a.

Let $\mathcal{G}^{\prime}$ be a Cauchy filter on $X^{\prime}$. Hence $\mathcal{G}^{\prime} \times \mathcal{G}^{\prime} \geqq \Phi^{\prime} \wedge\left[\Delta_{1}\right]$ for some $\Phi=\Phi^{-1} \in I$ and $\Phi \leqq[\Delta]$. Also, assume w.l.o.g. that $\mathcal{G}^{\prime}$ is not a fixed ultrafilter. Let $\Phi^{\prime}\left(\mathcal{S}^{\prime}\right)$ be the filter on $X^{\prime}$ generated by $\left\{A^{\prime}\left(G^{\prime}\right): A \in \Phi\right.$, $\left.G^{\prime} \in \mathcal{G}^{\prime}\right\}$, where $A^{\prime}\left(G^{\prime}\right)=\left\{\mathfrak{H C} \in X^{\prime}:(\mathcal{F}, \mathcal{H C}) \in A^{\prime}\right.$ for some $\left.\mathfrak{F} \in G^{\prime}\right\}$.

Lemma 2.4. The trace of $\Phi^{\prime}\left(\mathcal{G}^{\prime}\right)$ on $i(X)$ is a proper filter on $i(X)$.

LEMma 2.5. The trace of $\Phi^{\prime}\left(\mathcal{G}^{\prime}\right)$ on $X$ (recall $X$ and $i(X)$ are uniformly isomorphic) is an element of $X^{\prime}$.

Proof. Show that $\operatorname{Tr}_{X}\left(\Phi^{\prime}\left(\mathcal{G}^{\prime}\right)\right) \times \operatorname{Tr}_{X}\left(\Phi^{\prime}\left(\mathcal{G}^{\prime}\right)\right) \geqq \Phi^{3}$.

Lemma 2.6. The filter $\mathcal{G}^{\prime}$ converges in $\left(X^{\prime}, I^{\prime}\right)$ to the trace of $\Phi^{\prime}\left(\mathcal{G}^{\prime}\right)$ on $X$.

Proof. Show that $\mathcal{G}^{\prime} \times \phi\left(\operatorname{Tr}_{X}\left(\Phi^{\prime}\left(\mathcal{G}^{\prime}\right)\right)\right) \geqq\left(\Phi^{4}\right)^{\prime}$, where $\phi\left(\operatorname{Tr}_{X}\left(\phi^{\prime}\left(\mathcal{G}^{\prime}\right)\right)\right)$ is the fixed ultrafilter on $X^{\prime}$ generated by the set $\left\{\operatorname{Tr}_{X}\left(\Phi^{\prime}\left(\mathcal{G}^{\prime}\right)\right)\right\}$.

3. Hausdorff completions. Let $(X, I)$ be a u.c.s. and suppose $F$ converges to $x$; then $i(F)$ converges to $\phi(x)$ on $\left(X^{\prime}, I^{\prime}\right)$. Also $i(F)$ $\times \phi(F) \geqq(F \times F)^{\prime}$ and hence $i(F)$ also converges to $\mathcal{F}$. Consequently 
$\left(X^{\prime}, I^{\prime}\right)$ is in general not Hausdorff, even when $(X, I)$ is Hausdorff. We would like to identify $\mathfrak{F}$ and $\phi(x)$. The equivalence relation in [3] does just that. That is, $\mathcal{F} \sim \mathcal{G}$ iff $\mathcal{F} \wedge \mathcal{G}$ is Cauchy on $(X, I)$, which is shown to be equivalent to $\mathscr{F} \times \mathcal{G} \in I$. Hence if $\mathcal{F}$ converges to $x$, then $\mathcal{F} \sim \mathcal{G}$ iff $\mathcal{G}$ converges to $x$, which was desired.

The condition which is needed on a Hausdorff u.c.s. $(X, I)$ to construct a Hausdorff completion is the c.a., that is, $(X, I)$ satisfies the c.a. iff there exists a base $B$ for $I$ consisting of symmetric filters coarser than the diagonal filter such that $F \times F \geqq \Phi$ for each $\Phi$ in $B$, where $\mathcal{F}$ is any Cauchy filter on $(X, I)$.

Let $X^{*}$ be the quotient set $X^{\prime} / \sim$ with $P: X^{\prime} \rightarrow X^{*}$ being the quotient map, i.e. $P(\mathfrak{F})=[\mathfrak{F}]$, where $[\mathfrak{F}]$ is the equivalence class containing $\mathcal{F}$. Denote by $B^{*}=\left\{P\left(\Phi^{\prime}\right): \Phi \in I\right\}$.

Lemma 3.1. If $(X, I)$ satisfies the c.a. and $\Phi \in B$ with ([F], [G]) $\in P\left(A^{\prime}\right), A \in \Phi$, then $\mathcal{F}$ and $\mathcal{G}$ are $A^{3}$-near $\left(\right.$ i.e. $\left.(\mathcal{F}, \mathcal{G}) \in\left(A^{3}\right)^{\prime}\right)$.

Proof. Since $([\mathcal{F}],[\mathcal{G}]) \in P\left(A^{\prime}\right)$, then there exists $\mathcal{F}_{1} \sim \mathcal{F}$ and $\mathcal{G}_{1} \sim \mathcal{G}$ such that $\mathcal{F}_{1}$ and $\mathcal{G}_{1}$ are $A$-near. Also $\mathfrak{F}_{1} \sim \mathcal{F}_{\text {implies }} \mathfrak{F} \times \mathcal{F}_{1} \geqq\left(\mathfrak{F} \wedge \mathcal{F}_{1}\right)$ $\times\left(\mathcal{F} \wedge \mathcal{F}_{1}\right) \geqq \Phi$ and hence $\mathcal{F}$ and $\mathcal{F}_{1}$ are $A$-near. Similarly, $\mathcal{G}$ and $\mathcal{G}_{1}$ are $A$-near. Since $\mathfrak{F} \times \mathcal{G}=\left(\mathfrak{F} \times \mathcal{F}_{1}\right) \circ\left(\mathcal{F}_{1} \times \mathcal{G}_{1}\right) \circ\left(\mathcal{G}_{1} \times \mathcal{G}\right)$, then $\mathcal{F}$ and $\mathcal{G}$ are $A^{3}$-near.

Lemma 3.2. Let $(X, I)$ satisfy the c.a. Then $B^{*}$ is a base for a u.c.s. $I^{*}$ for $X^{*}$.

Proof. The first three conditions of Proposition 2.2 are immediate. From Lemma 3.1, $P\left(\Phi^{\prime}\right) \circ P\left(\Phi^{\prime}\right) \geqq P\left(\left(\Phi^{6}\right)^{\prime}\right)$ and hence the lemma follows.

TheOREM 3.3. Let $(X, I)$ be Hausdorff and satisfy the c.a., then $\left(X^{*}, I^{*}\right)$ is a Hausdorff completion of $(X, I)$.

The proof will follow from our succeeding results. As before, it is straightforward to show that $P$ restricted to $i(X)$ is a uniform isomorphism onto its image. Hence $P \circ i$ is a uniform isomorphism from $(X, I)$ onto its image.

Proposition 3.4. Let $(X, I)$ be Hausdorff and satisfy the c.a. Then $\left(X^{*}, I^{*}\right)$ is Hausdorff.

Proof. From [3], it suffices to show that $\left(X^{*}, I^{*}\right)$ is $T_{1}$. Let $\phi([\mathcal{F}])$ denote the filter on $X^{*}$ generated by $\{[\mathcal{F}]\}$. If $\phi([\mathfrak{F}]) \times \phi([\mathcal{G}]) \in I^{*}$, then $\phi([\mathcal{F}]) \times \phi([\mathcal{G}]) \geqq P\left(\Phi^{\prime}\right)$ for some $\Phi \in B$. It suffices to show that $\mathfrak{F} \times \mathcal{G} \geqq \Phi^{3}$. If $A \in \Phi$, then ([F], [G]) $\in P\left(A^{\prime}\right)$ and by Lemma 3.1, $\mathfrak{F}$ and $\mathcal{G}$ are $A^{3}$-near. Hence $[\mathcal{F}]=[\mathcal{G}]$ and the proposition follows. 
Let $[\mathfrak{F}] \in X^{*}$; then since $i(\mathfrak{F})$ converges to $\mathcal{F}, P(i(\mathfrak{F}))$ converges to $[\mathfrak{F}]$. Hence $(X, I)$ is dense in $\left(X^{*}, I^{*}\right)$.

Proposition 3.5. If $(X, I)$ satisfies the c.a., then $\left(X^{*}, I^{*}\right)$ is complete.

Proof. If $\mathcal{G}^{*}$ is a Cauchy filter on $\left(X^{*}, I^{*}\right)$, then $\mathcal{G}^{*} \times \mathcal{G}^{*} \geqq P\left(\Phi^{\prime}\right)$ for some $\Phi \in B$. Using Lemma 3.1, it is routine to show that $P^{-1}\left(\mathrm{G}^{*}\right)$ $\times P^{-1}\left(\mathcal{G}^{*}\right) \geqq\left(\Phi^{3}\right)^{\prime}$ and hence $P^{-1}\left(\mathcal{G}^{*}\right)$ is Cauchy on $\left(X^{\prime}, I^{\prime}\right)$. Since $\left(X^{\prime}, I^{\prime}\right)$ is complete, then $P^{-1}\left(\mathcal{G}^{*}\right)$ converges to $\mathcal{F}$ for some $\mathcal{F}$ in $X^{\prime}$. However, the continuity of $P$ implies that $\mathcal{G}^{*}$ converges to [F]. This concludes the proof of Theorem 3.3.

The remainder of this section will be devoted to showing that $\left(X^{*}, I^{*}\right)$ is in fact a natural generalization of the Bourbaki completion for Hausdorff uniform spaces.

If $(X, I)$ satisfies the c.a. and $\mathfrak{F} \in X^{\prime}$ with $\mathscr{F} \times \mathfrak{F} \geqq \Phi$, then as in uniform spaces [2], $\chi=\Phi(F)$, i.e. the filter on $X$ generated by $\{A(F): A \in \Phi, F \in \mathcal{F}\}$, is the unique minimal Cauchy filter on $X$ coarser than $\mathcal{F}$.

Let $Y$ denote the set of all minimal Cauchy filters on $(X, I)$ and $C=\left\{\Phi^{\prime \prime}: \Phi \in B, B\right.$ the c.a. base for $\left.(X, I)\right\}$, where $\Phi^{\prime \prime}$ is the filter on $Y \times Y$ generated by $\left\{A^{\prime \prime}: A \in \Phi\right\}$, and $A^{\prime \prime}=\left\{\left(\chi_{1}, \chi_{2}\right) \in Y \times Y\right.$ : $F \times F \subset A$ for some $\left.F \in \chi_{1} \wedge \chi_{2}\right\}$. As before, $C$ is a base for a u.c.s. $J$ for $Y$.

Theorem 3.6. If $(X, I)$ satisfies the c.a., then $\left(X^{*}, I^{*}\right)$ and $(Y, J)$ are uniformly isomorphic.

Proof. Define $j: X^{*} \rightarrow Y$ as follows: $j([\mathfrak{F}])=\chi$, where $\chi$ is the unique minimal Cauchy filter coarser than $\mathcal{F}$. Let $\mathcal{G} \sim \mathcal{F}$ and $\chi_{1}, \chi_{2}$ be the unique minimal Cauchy filters coarser than $\mathcal{G}$ and $\mathcal{F}$, respectively. Then $\chi_{1} \sim \mathcal{G} \sim \mathcal{F} \sim \chi_{2}$ and hence $\chi_{1} \wedge \chi_{2} \in X^{\prime}$. By the uniqueness of $\chi_{1}$ and $\chi_{2}$, we have that $\chi_{1}=\chi_{2}$ and thus $j$ is well defined. Clearly $j$ is a bijection. Also it can be shown that $j\left(P\left(\Phi^{\prime}\right)\right) \geqq\left(\Phi^{3}\right)^{\prime \prime}$ and $j^{-1}\left(\Phi^{\prime \prime}\right)$ $\geqq P\left(\Phi^{\prime}\right)$ for $\Phi \in B$. Hence $j$ is a uniform isomorphism.

4. Uniqueness among Hausdorff completions. Let $(X, I)$ be Hausdorff and satisfy the c.a. Then we will consider $(X, I)$ as a subspace of $\left(X^{*}, I^{*}\right)$.

Proposition 4.1. If $(X, I)$ is Hausdorff and satisfies the c.a., then any two Hausdorff completions of $(X, I)$ are equipotent sets.

Proof. Let $(Z, T)$ be a Hausdorff completion of $(X, I)$; then define $\alpha: Z \rightarrow X^{*}$ as follows: $\alpha$ is the identity map on $X$ and $\alpha(s)=[\mathcal{F}]$, 
where $\mathcal{F} \in X^{\prime}$ and $\mathcal{F}$ converges to $s$ on $(Z, T)$. It is straightforward to show that $\alpha$ is a bijection.

Lemma 4.2. If $(X, I)$ is Hausdorff and satisfies the c.a., then $P\left(\Phi^{\prime}\right)$ $\geqq \mathrm{Cl}_{X^{*} \times X^{*}}(\Phi) \geqq P\left(\left(\Phi^{3}\right)^{\prime}\right)$ for $\Phi \in B$.

Proof. The first inequality is straightforward. We will verify the latter. Let $A \in \Phi$ and $([\mathcal{F}],[\mathcal{G}]) \in \mathrm{Cl} A$. Hence there exists $\mathfrak{F}^{*}, \mathfrak{K}^{*}$ on $X^{*}$ such that $\mathfrak{H}^{*}$ converges to $[\mathfrak{F}], \mathfrak{K}^{*}$ converges to [S], where $\mathfrak{H}^{*} \times \mathcal{K}^{*}$ and $A$ are not disjoint. Let $\mathcal{H}^{\mathcal{C}}=\operatorname{Tr}_{X} \mathcal{H}^{*}, \mathcal{K}=\operatorname{Tr}_{X} \mathcal{K}^{*}$. By the c.a., $\mathcal{H} \times \mathfrak{H} \geqq \Phi$ and $\mathscr{K} \times \mathfrak{K} \geqq \Phi$. Hence $H \times K=(H \times H) \circ A \circ$ $(K \times K) \subset A^{3}$ for some $H \in \mathfrak{H}, K \in \mathcal{K}$. Thus $(\mathfrak{H C}, \mathfrak{K}) \in\left(A^{3}\right)^{\prime}$, or $([\mathfrak{H C}$, $[\mathscr{K}]) \in P\left(\left(A^{3}\right)^{\prime}\right)$. Since $\mathfrak{F}^{*}$ converges to $[\mathcal{F}]$, then $\mathfrak{F}$ converges to $[\mathfrak{F}]$; however, $\mathfrak{H}$ also converges to $[\mathfrak{H C}$. Hence $[\mathfrak{F}]=[\mathfrak{F C}]$ and similarly $[\mathcal{G}]=[\mathfrak{K}]$.

Proposition 4.3. Let $(X, I)$ be Hausdorff and satisfy the c.a. with base $B$. If $f:(X, I) \rightarrow(Z, T)$ is uniformly continuous with $(Z, T)$ being Hausdorff, complete and such that $\mathrm{Cl} f(\Phi)$ belongs to $T$ for each $\Phi \in B$, then $f$ has a unique uniformly continuous extension $g$ to $\left(X^{*}, I^{*}\right)$.

Proof. This follows from a routine argument showing that $g\left(P\left(\Phi^{\prime}\right)\right) \geqq \operatorname{Cl} f(\Phi)$.

THEOREM 4.4. Let $(X, I)$ be Hausdorff and satisfy the c.a. with base $B$. If $(Z, T)$ is a Hausdorff completion of $(X, I)$, then the identity map extends to a uniform isomorphism between $\left(X^{*}, I^{*}\right)$ and $(Z, T)$ iff $B_{1}$ $=\left\{\mathrm{Cl}_{Z_{\times} Z} \Phi: \Phi \in B\right\}$ is a base for $T$.

Proof. Let $\Phi \in B$; then if $g$ is the extension of $i$ to $\left(X^{*}, I^{*}\right)$, $g\left(\mathrm{Cl}_{X^{*} \times X^{*}} \Phi\right) \geqq \mathrm{Cl}_{Z \times Z} \Phi$. If $A \in \Phi$ with $(s, t) \in \mathrm{Cl}_{Z \times Z} A$, then there exists $\mathcal{F}, \mathcal{G}$ on $X$ such that $\mathcal{F}$ and $\mathcal{G}$ converge to $s$ and $t$, respectively, and $\mathfrak{F} \times \mathcal{G}$ is not disjoint from $A$. Hence $[\mathfrak{F}],[\mathcal{G}] \in X^{*}$ and since $\mathfrak{F}, \mathcal{G}$ converge to $[\mathcal{F}]$ and $[\mathcal{G}]$, respectively on $\left(X^{*}, I^{*}\right)$ then $([\mathcal{F}],[\mathcal{G}])$ $\in \mathrm{Cl}_{X^{*} \times X^{*}} A$. Thus $g\left(\mathrm{Cl}_{X^{*} \times X^{*}} \Phi\right)=\mathrm{Cl}_{Z \times Z} \Phi$ and from Lemma 4.2, $B_{1}$ is a base for $T$.

Conversely, let $\alpha$ be as in the proof of Proposition 4.1. If $\Phi \in B$, then it is straightforward to show that $\alpha(\mathrm{Cl} \Phi) \geqq \mathrm{Cl} \Phi^{3}$. Hence $\alpha$ is uniformly continuous and $\alpha^{-1}$ is the extension of the identity map.

COROllary 4.5 (Bourbaki). Every Hausdorff uniform space has precisely one Hausdorff completion in the uniform space sense (up to a uniform isomorphism).

Proof. From Theorem 3.6, $\left(X^{*}, I^{*}\right)$ is uniformly isomorphic to the Bourbaki completion. Also the hypothesis of the sufficiency of The- 
orem 4.4 is satisfied (p. 178 of [2]) and hence the corollary follows.

In [5] it is shown that a u.c.s. which satisfies the c.a. induces a regular topology. It would be interesting to know just how much, if any the hypothesis that the space satisfy the c.a. could be relaxed and still obtain a completion.

\section{REFERENCES}

1. H. J. Biesterfeldt, Jr., Completion of a class of uniform convergence spaces, Nederl. Akad. Wetensch. Proc. Ser. A69=Indag. Math. 28 (1966), 602-604. MR 34 \#5052.

2. N. Bourbaki, General topology, Part I, Hermann, Paris; Addison-Wesley, Reading, Mass., 1966. MR 34 \#5044a.

3. C. H. Cook and H. R. Fischer, Uniform convergence structures, Math. Ann. 173 (1967), 290-306. MR 36 \#845.

4. H. R. Fischer, Limesräume, Math. Ann. 137 (1959), 269-303. MR 22 \#225.

5. G. D. Richardson, A class of uniform convergence structures, Proc. Amer. Math. Soc. 25 (1970), 399-402.

North Carolina State University, Raleigh, North Carolina 27607 